\section{BMJ Open Respiratory Research}

\title{
SARS-CoV-2 organising pneumonia: 'Has there been a widespread failure to identify and treat this prevalent condition in COVID-19?'
}

\author{
Pierre Kory (D) , ${ }^{1}$ Jeffrey P Kanne ${ }^{2}$
}

To cite: Kory P, Kanne JP. SARS-CoV-2 organising pneumonia: 'Has there been a widespread failure to identify and treat this prevalent condition in COVID-19?'. BMJ Open Resp Res 2020;7:e000724. doi:10.1136/ bmjresp-2020-000724

Received 20 July 2020 Revised 27 August 2020 Accepted 5 September 2020
Check for updates

(C) Author(s) (or their employer(s)) 2020. Re-use permitted under CC BY-NC. No commercial re-use. See rights and permissions. Published by BMJ.

${ }^{1}$ Advocate Aurora Critical Care Service, Aurora St Luke's Medical Center, Milwaukee, Wisconsin, USA

${ }^{2}$ School of Medicine and Public Health, University of Wisconsin-Madison, Madison, Wisconsin, USA

Correspondence to Dr Pierre Kory; pierrekory@icloud.com

\section{ABSTRACT}

Reviews of COVID-19 CT imaging along with postmortem lung biopsies and autopsies indicate that the majority of patients with COVID-19 pulmonary involvement have secondary organising pneumonia (OP) or its histological variant, acute fibrinous and organising pneumonia, both well-known complications of viral infections. Further, many publications on COVID-19 have debated the puzzling clinical characteristics of 'silent hypoxemia', 'happy hypoxemics' and 'atypical ARDS', all features consistent with OP. The recent announcement that RECOVERY, a randomised controlled trial comparing dexamethasone to placebo in COVID-19, was terminated early due to excess deaths in the control group further suggests patients present with OP given that corticosteroid therapy is the first-line treatment. Although RECOVERY along with other cohort studies report positive effects with corticosteroids on morbidity and mortality of COVID-19, treatment approaches could be made more effective given that secondary OP often requires prolonged duration and/or careful and monitored tapering of corticosteroid dose, with 'pulse' doses needed for the well-described fulminant subtype. Increasing recognition of this diagnosis will thus lead to more appropriate and effective treatment strategies in COVID-19, which may lead to a further reduction of need for ventilatory support and improved survival.

\section{INTRODUCTION}

From the earliest reports of the novel virus SARS-CoV-2 causing a respiratory illness (COVID-19), clinicians remarked on the puzzling discordance between the degree of hypoxemia and relatively modest work of breathing observed. Early reports described this combination as 'silent hypoxemia' and such patients as 'happy hypoxemics'. ${ }^{2}$ Similarly, soon after mechanical ventilation was instituted, unexpectedly high degrees of lung compliance in conjunction with severe hypoxemia was deemed a new ' $L$ ' phenotype of acute hypoxemic respiratory failure, attributed to an early phase 'dry lung' with measured 'hyperperfusion of gasless tissue' as opposed to the significant alveolar oedema and resulting hypoxic vasoconstriction observed in 'traditional' acute respiratory distress syndrome (ARDS). ${ }^{3}$
We believe that clinicians have not sufficiently considered the condition of 'viral-induced secondary organising pneumonia (OP)', largely due to the fact that OP in its idiopathic form, called cryptogenic organising pneumonia $(\mathrm{COP})$, is a rare and clinically unique disease often misunderstood and poorly recognised even by pulmonologists. OP is a histological pattern of lung injury characterised by the filling of alveoli and alveolar ducts with spindleshaped fibroblasts and myofibroblasts that later form granulation tissue. ${ }^{4}$ However, OP more commonly results from infections (especially viral), drugs or autoimmunity, when the term secondary OP applies. ${ }^{5} \mathrm{COP}$ and secondary OP have indistinguishable clinical and radiographic findings, relapse rates, mortality and robust responses to corticosteroid therapy. ${ }^{5}$ Viralinduced OP during the severe acute respiratory syndrome (SARS), Middle East respiratory syndrome (MERS) and H1N1 viral pandemics have been well described. ${ }^{6}$ With SARS, OP and its histological variant, acute fibrinous and organising pneumonia (AFOP), were reported in $30 \%-60 \%$ of intensive care unit patients. The reported CT findings of COVID-19 suggest that secondary OP, AFOP or both may be occurring even more frequently. ${ }^{8-10}$

In this perspective, we review the clinical, radiographic, histopathological and treatment response characteristics supporting the conclusion that SARS-CoV-2-induced secondary $\mathrm{OP}$, AFOP or both is the underlying cause of COVID-19 respiratory disease in the majority of patients.

\section{CLINICAL PRESENTATIONS OF OP AND COVID-19}

OP has a unique clinical presentation generally recognised by only experienced clinicians. ${ }^{11}$ The widespread lack of familiarity with the clinical presentation of OP may explain the recent COVID-19 publication titles such as "Is COVID-19 typical ARDS?", "The mystery 
of the pandemic's 'happy hypoxia"" and "Why COVID-19 silent hypoxemia is baffling to physicians", including a pro-con debate where COVID-19 was theorised similar to high-altitude pulmonary oedema. ${ }^{12}{ }^{12}$ Although OP can present similarly to mild infectious pneumonia, inflammatory markers such as $\mathrm{C}$ reactive protein are often markedly elevated similar to COVID-19. ${ }^{11}$

The descriptions of patients with COVID-19 presenting with 'silent hypoxemia' (low oxygen levels in the absence of respiratory distress) are strikingly similar to descriptions of OP where one report stated "hypoxemia with alveolar right-to-left shunt may be well tolerated". ${ }^{11}$ Another description included "gas exchange abnormalities are extremely common with a reduction in the diffusing capacity of oxygen and resting hypoxemia being almost universal findings" and "OP typically presents with mild dyspnea although occasionally it may be severe, especially in the event of rapidly progressive disease, which more commonly occurs with AFOP". 114 More specifically, one study assessed over 1100 pre-hospital COVID-19 patients and found a significantly higher $\mathrm{Sp}_{2} /$ respiratory rate ratio compared with pre-COVID-19 patients, substantiating far lower respiratory rates per degree of hypoxemia than is typical for other causes of acute hypoxemia.

Thus, the clinical course of COVID-19 and secondary OP tend to follow a subacute respiratory illness, although in both conditions a rapid-onset progression to fulminant respiratory failure and even death from extensive fibrosis has been described, with such cases reported to occur in approximately $5 \%-8 \%$ of secondary OP. Such fulminant cases may be contributing to the US mortality rate ranges reported among COVID-19-infected patients prior to the recent adoption of corticosteroid therapy. ${ }^{14-16}$

\section{RADIOGRAPHIC EVIDENCE FOR OP IN COVID-19}

Both common and uncommon imaging patterns of OP have been described. ${ }^{15}$ However, in approximately $60 \%-90 \%$ of

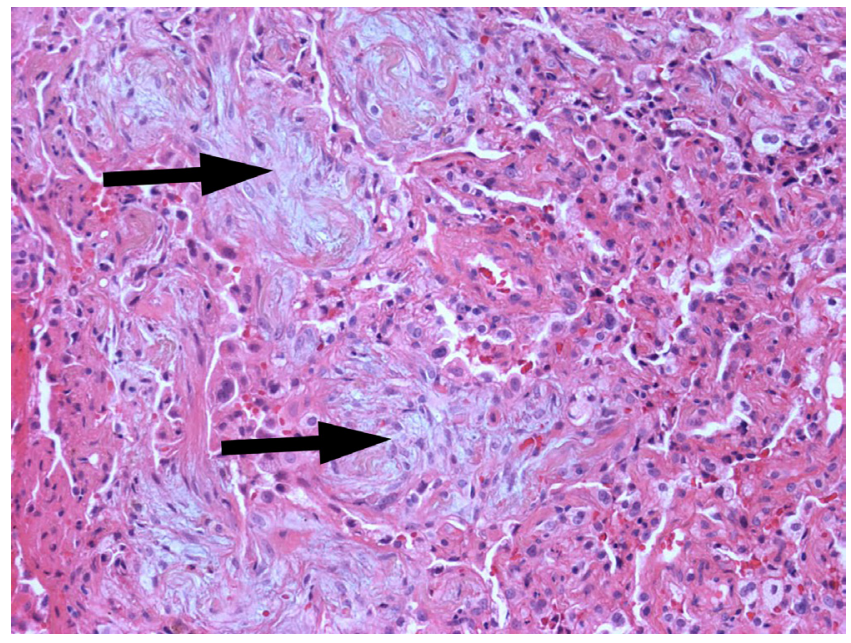

Figure 1 Photomicrograph shows immature collagen plugs or Masson bodies (arrows) filling the airspaces. There is mild chronic interstitial inflammation. (H\&E, medium power). patients with OP, the radiographic and CT findings are often so characteristic that they suggest the pattern of injury. ${ }^{15}$ The 'archetypal imaging findings' refer to (1) peripheral, bilateral, lower lung predominant consolidation or even a frequent appearance in all lungs zones and/or (2) peribronchovascular consolidation, which can extend to the subpleural regions in the lower lobes associated with patchy ground-glass opacities (GGO). ${ }^{15}$ The most compelling support for $\mathrm{OP}$ as the underlying pattern of lung injury from COVID-19 comes from an expert panel review, published in March 2020, which reported "the most common reported CT findings in COVID-19 patients are typical of an organizing pneumonia pattern of lung injury", with this pattern now described in virtually all published cases. ${ }^{8-10}$ Further, in a study of the diagnostic accuracy of CT among idiopathic interstitial pneumonias, the correct diagnosis of $\mathrm{COP}$ was the highest, in $79 \%$ of cases, supporting that CT imaging features are characteristic and that "the typical imaging features of COP are usually so characteristic that they allow the possibility of diagnosis for most experienced clinicians". ${ }^{17}$

Although variable radiographic findings in AFOP have been described, patients who experience a rapidly progressive course exhibit imaging findings similar to diffuse alveolar damage (DAD), with diffuse but basilar-predominant consolidation and GGO. ${ }^{18}$ Those with a more subacute course may have similar radiological findings of cryptogenic OP with focal or diffuse parenchymal abnormalities. ${ }^{18}$

\section{PATHOLOGICAL EVIDENCE FOR OP IN COVID-19}

Although myriad agents and organisms are capable of injuring the lung, the histological responses to acute injury generally take three forms: $\mathrm{DAD}$, OP or AFOP, and eosinophilic pneumonia (EP), with EP not yet described in COVID19. In DAD, 'diffuse' refers to injury of both the alveolar and endothelial cell layers, thus causing fluid and proteins to leak into and accumulate in the alveolus. The two prominent phenotypes are the acute/exudative and organising/ proliferative, the former referring to the early phase where the leaked proteins form hyaline membranes that line the walls of the alveolus and cause non-resolution of the oedema while the latter is characterised by resolution of the oedema with interstitial infiltration by myofibroblasts and early deposition of collagen. In some patients, this stage progresses to fibrosis characterised by obliteration of the lung architecture. In OP and AFOP, the alveolar epithelial injury causes leakage of coagulative proteins, which accumulate fibrin due to diminished fibrinolytic activity. In OP, fibroblast activation and proliferation then follow, producing a connective tissue matrix in the alveolus and ducts (figure 1). In AFOP, although similar 'organisation' occurs, a high degree of accumulated 'fibrin balls' are the dominant histological finding. ${ }^{19}$ Similarly, in DAD, after the acute/exudative phase, an organising/proliferative phase of DAD follows. Although hyaline membranes and oedema are still present, the interstitial location and proliferation of fibroblasts is the hallmark of this phase. The protein, oedema, fibrin or organisation 


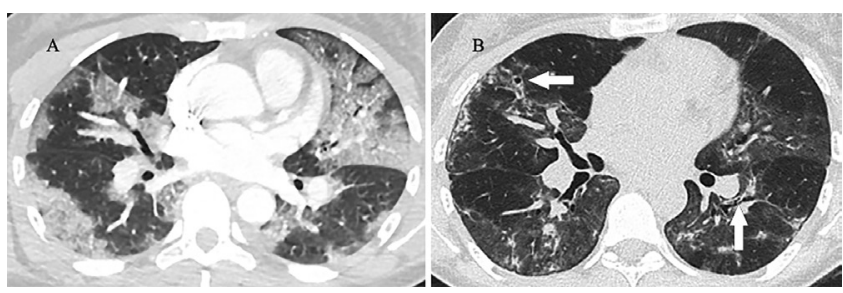

Figure 2 37-year-old woman with COVID-19 requiring $\mathrm{FiO}_{2}$ of 1.0 delivered via heated humidified high-flow nasal cannula for 8 days. On day 8 , initiation of treatment with 'pulse-dose' methylprednisolone $1000 \mathrm{mg}$ intravenously for 3 days was followed by an abrupt reduction in oxygen needs and eventual discharge on ambient air. (A) Contrast-enhanced thin-section CT image 6 days after onset of symptoms shows peripheral and peri-bronchial ground-glass opacity in both lungs typical of an organising pneumonia pattern of lung injury. (B) Unenhanced CT image 2 weeks after discharge shows marked clearing of groundglass opacity with small foci of lung consolidation and minimal traction bronchiectasis (arrows) suggesting mild fibrosis.

foci of DAD, AFOP and OP can resolve or result in permanent pulmonary fibrosis.

Initially, AFOP was considered a variant of DAD due to a similar aggressive behaviour and mortality rate; however, more recent studies have found that the clinical course and prognosis of AFOP are better than and closer to those of OP. Some lung pathologists have argued that AFOP is a histological variant of OP, yet this is not a uniformly accepted categorisation thus AFOP and OP are likely better understood as distinct entities that may occur from similar insults and respond to similar therapies. Further, they both can follow a fulminant course. ${ }^{1920}$

The histological pattern of AFOP in patients with COVID-19 was reported in a series of postmortem lung biopsies performed on six patients with COVID-19. ${ }^{21}$ The histopathological pattern in the five patients who died approximately 20 days after the onset of symptoms was AFOP. Although AFOP was diagnosed, the uncertain amount of tissue examined may have missed the presence of hyaline membranes which would have supported the diagnosis of DAD. Another report of five autopsies in patients with severe COVID-19 also found significant fibrin accumulation with microvascular injury without evidence of the hyaline membranes define the diagnosis of DAD. ${ }^{22}$ A recent systematic review of all pathological findings in COVID-19 reported that $59 \%$ of specimens had findings of microvascular injury, AFOP or both, often coexisting with 'epithelial' injury (DAD) or 'fibrosis' ${ }^{23}$ Although the patterns could coexist, the fibrotic pattern was not seen until at least 3 weeks from symptoms and was thought to represent fibrosing OP in at least one case.

Unfortunately, to date, no tissue biopsies have been performed ante mortem in the early phase of disease; however, one autopsy study of 12 subjects who died within the first 14 days after onset of symptoms (five patients died within 1 week of symptom onset) reported "interestingly, the 'organizing phase' of DAD was observed in patients with a brief symptom onset-to-death interval, which is in contrast to SARS where it was predominantly observed in those with longer hospitalizations". ${ }^{24}$ The authors thought it surprising that the 'organising' phase of DAD was present so soon after symptom onset, and posited that the 'exudative' phase of DAD must have been subclinical given that asymptomatic patients all had 'abnormal CT findings' as reported in a paper which compiled CT findings from a cohort of patients who were pre-symptomatic. ${ }^{25}$ However, the CT findings in this cohort were all consistent with $\mathrm{OP}$ and not DAD, thus suggesting that OP was likely the initial response to injury, with DAD then occurring later perhaps as a result of a thrombotic microangiopathy, cytopathic effect or the subsequent ventilator-induced lung injury, of which DAD is the hallmark. This progression is further supported by the fact that OP is known to present with radiographic abnormalities despite minimal or no symptoms. ${ }^{19}$

Ultimately, the exact sequence of progression is currently unknown due to the lack of early, antemortem tissue biopsy in patients with COVID-19 along with the inherent bias that results from classifying COVID-19 lung injury only among those most severely affected given that the majority of autopsies were performed in patients after prolonged mechanical ventilation which unsurprisingly demonstrated DAD. Thus, in the early phases of the disease, the radiological findings and clinical assessment should be weighed more heavily in determining the type of lung injury occurring. The exact prevalence of OP or AFOP will ultimately be determined over time as the body of postmortem (and potentially antemortem) pathological studies accumulate.

\section{THERAPEUTIC RESPONSE TO CORTICOSTEROIDS IN OP AND COVID-19}

Corticosteroid therapy is the first-line treatment for both OP and AFOP and often results in proximate improvements in symptoms, radiographic findings and need for oxygen (figure 2). ${ }^{18}$ Recently, the RECOVERY trial reported dramatic mortality reductions when dexamethasone was initiated early in the treatment of COVID-19 hospitalised patients requiring oxygen or mechanical ventilation. ${ }^{26}$ These results are consistent with and add validation to multiple recently published randomised and cohort studies from Italy, Spain and the USA showing an association of corticosteroid treatment with decreased mortality and need for ventilators. $^{27-29}$

\section{CONCLUSION}

It is our view that, based on the similar clinical presentations, radiographic abnormalities, overlapping yet supportive histopathological patterns on autopsy in conjunction with studies reporting that patients are 'steroid-responsive', early COVID-19 respiratory disease is better understood primarily as 'SARS-CoV-2 induced secondary OP'.

Given this likely high prevalence of OP, AFOP or both in early COVID-19, a concern is that the increasingly adopted RECOVERY trial protocol $(6 \mathrm{mg}$ dexamethasone daily for up to 10 days) may be insufficient given that treatment of 
secondary OP often requires higher doses, prolonged duration of treatment, and a careful and monitored tapering. ${ }^{9}$ Thus, additional studies comparing corticosteroid type, dosing and duration should be conducted along with the use of other immunosuppressive agents. Initial and maintenance corticosteroid dosing should be similar to that recommended to treat COP, although patients with secondary OP typically require a shorter duration. ${ }^{30}$ Clinicians should also be aware of the higher 'pulse' doses required in the successful treatment of fulminant cases of OP or AFOP. Although chronic macrolide therapy has demonstrated efficacy as a steroid-sparing agent in the treatment of COP, there are insufficient data to support a recommendation for use in COVID-19 secondary OP. ${ }^{31-33}$

Contributors PK originally developed the hypothesis, wrote the first draft of the majority of the manuscript with the exception of the radiological literature review and summary interpretation of the review. He also compiled the references and figure and prepared the final version of the manuscript. JPK contributed to the development of the main hypothesis, wrote portions of the manuscript related to the review of the radiographic literature as well as provided radiographic interpretations as well as provided several key radiology literature references and also completed and refined the final version of the figure. He was also instrumental in final review and editing of the manuscript.

Funding The authors have not declared a specific grant for this research from any funding agency in the public, commercial or not-for-profit sectors.

Competing interests None declared.

Patient consent for publication Obtained.

Provenance and peer review Not commissioned; externally peer reviewed.

Open access This is an open access article distributed in accordance with the Creative Commons Attribution Non Commercial (CC BY-NC 4.0) license, which permits others to distribute, remix, adapt, build upon this work non-commercially, and license their derivative works on different terms, provided the original work is properly cited, appropriate credit is given, any changes made indicated, and the use is non-commercial. See: http://creativecommons.org/licenses/by-nc/4.0/.

ORCID iD

Pierre Kory http://orcid.org/0000-0002-0816-9682

\section{REFERENCES}

1 Tobin MJ, Laghi F, Jubran A. Why COVID-19 silent hypoxemia is baffling to physicians. Am J Respir Crit Care Med 2020;202:356-60.

2 Couzin-Frankel J. The mystery of the pandemic's 'happy hypoxia'. Science 2020;368:455-6.

3 Gattinoni L, Chiumello D, Caironi P, et al. COVID-19 pneumonia: different respiratory treatments for different phenotypes? Intensive Care Med 2020;46:1099-102.

4 Kligerman SJ, Franks TJ, Galvin JR. From the radiologic pathology archives: organization and fibrosis as a response to lung injury in diffuse alveolar damage, organizing pneumonia, and acute fibrinous and organizing pneumonia. Radiographics 2013;33:1951-75.

5 Drakopanagiotakis F, Paschalaki K, Abu-Hijleh M, et al. Cryptogenic and secondary organizing pneumonia: clinical presentation, radiographic findings, treatment response, and prognosis. Chest 2011;139:893-900.

6 Cornejo R, Llanos $\mathrm{O}$, Fernández $\mathrm{C}$, et al. Organizing pneumonia in patients with severe respiratory failure due to novel $A(\mathrm{H} 1 \mathrm{~N} 1)$ influenza. BMJ Case Rep 2010;2010:bcr0220102708-5.

7 Hwang DM, Chamberlain DW, Poutanen SM, et al. Pulmonary pathology of severe acute respiratory syndrome in Toronto. Mod Pathol 2005;18:1-10.

8 Kanne JP, Little BP, Chung JH, et al. Essentials for radiologists on COVID-19: an update-Radiology Scientific Expert Panel. Radiology 2020;296:E113-4.
9 Fu F, Lou J, Xi D, et al. Chest computed tomography findings of coronavirus disease 2019 (COVID-19) pneumonia. Eur Radiol 2020;91.

10 Hani C, Trieu NH, Saab I. Elsevier has created a COVID-19 resource centre with free information in English and Mandarin on the novel coronavirus COVID-19. The COVID-19 resource centre is hosted on Elsevier Connect, the company's public news and information, 2020.

11 Cordier J-F. Cryptogenic organising pneumonia. Eur Respir J 2006;28:422-46.

$12 \mathrm{Li} \mathrm{X,} \mathrm{MaX.} \mathrm{Acute} \mathrm{respiratory} \mathrm{failure} \mathrm{in} \mathrm{COVID-19:} \mathrm{is} \mathrm{it} \mathrm{"typical"}$ ARDS? Crit Care 2020;24:1-5.

13 Kyle-Sidell C. COVID-19 lung injury and "typical" ARDS: the danger of presumed equivalency. Ann Am Thorac Soc 2020;0:2-5.

14 King TE, Mortenson RL. Cryptogenic organizing pneumonitis. The North American experience. Chest 1992;102:8S-13.

15 Torrealba JR, Fisher S, Kanne JP, et al. Pathology-radiology correlation of common and uncommon computed tomographic patterns of organizing pneumonia. Hum Pathol 2018;71:30-40.

16 NYC Department of Health. COVID-19:data. Available: https:// www1.nyc.gov/site/doh/COVID/COVID-19-data.page

17 Johkoh T, Müller NL, Cartier Y, et al. Idiopathic interstitial pneumonias: diagnostic accuracy of thin-section CT in 129 patients. Radiology 1999;211:555-60.

$18 \mathrm{Kim}$ JY, Doo KW, Jang H-J. Acute fibrinous and organizing pneumonia: Imaging features, pathologic correlation, and brief literature review ${ }^{2}$. Radiol Case Rep 2018;13:867-70.

19 Feinstein MB, DeSouza SA, Moreira AL, et al. A comparison of the pathological, clinical and radiographical, features of cryptogenic organising pneumonia, acute fibrinous and organising pneumonia and granulomatous organising pneumonia. J Clin Pathol 2015;68:441-7.

20 Dai J-H, Li H, Shen W, et al. Clinical and radiological profile of acute fibrinous and organizing pneumonia: a retrospective study. Chin Med J 2015;128:2701-6.

21 Copin M-C, Parmentier E, Duburcq T, et al. Time to consider histologic pattern of lung injury to treat critically ill patients with COVID-19 infection. Intensive Care Med 2020;46:1124-6.

22 Magro C, Mulvey JJ, Berlin D, et al. Complement associated microvascular injury and thrombosis in the pathogenesis of severe COVID-19 infection: a report of five cases. Transl Res 2020;220:1-13.

23 Polak SB, Van Gool IC, Cohen D, et al. A systematic review of pathological findings in COVID-19: a pathophysiological timeline and possible mechanisms of disease progression. Modern Pathol 2020;579.

24 Bradley BT, Maioli $\mathrm{H}$, Johnston R, et al. Histopathology and ultrastructural findings of fatal COVID-19 infections. medRxiv 2020.

25 Shi H, Han X, Jiang N, et al. Radiological findings from 81 patients with COVID-19 pneumonia in Wuhan, China: a descriptive study. Lancet Infect Dis 2020.

26 Horby P, Lim WS, Emberson J, et al. Effect of dexamethasone in hospitalized patients with COVID-19: preliminary report. medRxiv 2020.

27 Corral L, Bahamonde A, delas RFA, et al. GLUCOCOVID: a controlled trial of methylprednisolone in adults hospitalized with COVID-19 pneumonia. medRxiv 2020.

28 Fadel R, Morrison AR, Vahia A, et al. Early short course corticosteroids in hospitalized patients with COVID-19. Clin Infect Dis 2020. doi:10.1093/cid/ciaa601. [Epub ahead of print: 19 May 2020].

29 Salton F, Confalonieri P, Santus P, et al. Prolonged low-dose methylprednisolone in patients with severe COVID-19 pneumonia. medRxiv 2020.

30 Basarakodu KR, Aronow WS, Nair CK, et al. Differences in treatment and in outcomes between idiopathic and secondary forms of organizing pneumonia. Am J Ther 2007;14:422-6.

31 Ding Q-L, Lv D, Wang B-J, et al. Macrolide therapy in cryptogenic organizing pneumonia: a case report and literature review. Exp Ther Med 2015;9:829-34.

32 Rosenberg ES, Dufort EM, Udo T, et al. Association of treatment with hydroxychloroquine or azithromycin with in-hospital mortality in patients with COVID-19 in New York state. JAMA 2020;323:2493.

33 Cavalcanti AB, Zampieri FG, Rosa RG, et al. Hydroxychloroquine with or without azithromycin in mild-to-moderate Covid-19. N Engl $J$ Med 2020. doi:10.1056/NEJMoa2019014. [Epub ahead of print: 23 Jul 2020]. 